\title{
Revealing the most disturbing tendency error of Zebiak-Cane model associated with EI Niño predictions by nonlinear forcing singular vector approach
}

\author{
Wansuo Duan · Peng Zhao
}

Received: 19 December 2013 / Accepted: 9 October 2014 / Published online: 30 October 2014

(C) The Author(s) 2014. This article is published with open access at Springerlink.com

\begin{abstract}
The nonlinear forcing singular vector (NFSV) approach is used to identify the most disturbing tendency error of the Zebiak-Cane model associated with El Niño predictions, which is most potential for yielding aggressively large prediction errors of El Niño events. The results show that only one NFSV exists for each of the predictions for the predetermined model El Niño events. These NFSVs cause the largest prediction error for the corresponding El Niño event in perfect initial condition scenario. It is found that the NFSVs often present largescale zonal dipolar structures and are insensitive to the intensities of El Niño events, but are dependent on the prediction periods. In particular, the NFSVs associated with the predictions crossing through the growth phase of El Niño tend to exhibit a zonal dipolar pattern with positive anomalies in the equatorial central-western Pacific and negative anomalies in the equatorial eastern Pacific (denoted as "NFSV1"). Meanwhile, those associated with the predictions through the decaying phase of El Niño are inclined to present another zonal dipolar pattern (denoted as "NFSV2"), which is almost opposite to the NFSV1. Similarly, the linear forcing singular vectors (FSVs), which are computed based on the tangent linear model, can also be classified into two types "FSV1" and "FSV2". We find that both FSV1 and NFSV1 often cause negative prediction errors for Niño-3 SSTA of the El Niño events, while the FSV2 and NFSV2 usually yield positive
\end{abstract}

\footnotetext{
W. Duan $(\triangle) \cdot$ P. Zhao

LASG, Institute of Atmospheric Physics, Chinese Academy of Sciences, Beijing 100029, China

e-mail: duanws@lasg.iap.ac.cn

P. Zhao

China Meteorological Administration Training Center,

Beijing 100081, China
}

prediction errors. However, due to the effect of nonlinearities, the NFSVs usually have the western pole of the zonal dipolar pattern much farther west, and covering much broader region. The nonlinearities have a suppression effect on the growth of the prediction errors caused by the FSVs and the particular structure of the NFSVs tends to reduce such suppression effect of nonlinearities, finally making the NFSV-type tendency error yield much large prediction error for Niño-3 SSTA of El Niño events. The NFSVs, compared to the FSVs, are more applicable in describing the most disturbing tendency error of the Zebiak-Cane model since they consider the effect of nonlinearities. The NFSV-type tendency errors may provide information concerning the sensitive areas where the model errors are much more likely to yield large prediction errors for El Niño events. If the simulation skills of the states in the sensitive areas can be improved, the ENSO forecast skill may in turn be greatly increased.

Keywords Predictability · Model error · Optimal perturbation $\cdot$ El Niño event

\section{Introduction}

The El Niño-Southern Oscillation (ENSO) cycle has attracted the attention of scientists in recent decades because its environmental and socioeconomic impacts are felt worldwide (e.g., McPhaden et al. 2006). Knowledge of the ENSO cycle and forecasts of its variations are therefore valuable for agriculture, public health and safety, and many other climate-sensitive human endeavors.

Since the development of the Zebiak-Cane model (Zebiak and Cane 1987), which for the first time demonstrated the possibility of ENSO prediction by forecasting 
the 1986/1987 El Niño event in real time, a suite of models with varying degrees of complexity have been developed for ENSO modeling and prediction (Neelin 1990; Kleeman 1991; Latif et al. 1993; Penland and Magorian 1993; Luo et al. 2008). The climate forecast system at the National Centers for Environmental Prediction (NCEP) (Saha et al. 2006), the seasonal forecast systems at the European Center for Medium-Range Weather Forecasts (ECMWF), and the multi-model ensemble (MME) systems of the EU (Palmer et al. 2004) and the Asia-Pacific Economic Cooperation (APEC) Climate Center (APCC) have also been developed for seasonal to interannual climate prediction.

While significant progress has been made with respect to ENSO theories and predictions over the years, there still exist considerable errors when predicting ENSO events (Jin et al. 2008; Tang et al. 2008), possibly due to the uncertainty in initial conditions and model parameters, the inherent nonlinearity of ENSO, atmospheric noise, and other high-frequency variations. Many studies have explored ENSO predictability associated with prediction errors from the viewpoint of initial error growth (Moore and Kleeman 1996; Samelson and Tziperman 2001) and showed that initial errors may have a significant effect on ENSO predictions. Moore and Kleeman (1996) demonstrated the season-dependent evolutions of initial errors of ENSO events and illustrated the "spring predictability barrier" (SPB) phenomenon from the viewpoint of error growth. Chen et al. (1995) reduced the SPB phenomenon of the model developed by Zebiak and Cane (1987) through improving the initialization, and the result showed an enhancement of the ENSO forecasting skill. Recently, $\mathrm{Mu}$ et al. (2007a, b) further emphasized the importance of a particular initial error pattern in yielding the SPB for ENSO events.

In realistic ENSO predictions, the prediction errors are generally caused by initial errors and model errors. Furthermore, an increasing number of studies have indicated that model errors also influence the ability to forecast ENSO (Wu et al. 1993; Hao and Ghil 1994; Blanke et al. 1997; Flügel and Chang 1998; Latif et al. 1998; Liu 2002; Zhang et al. 2003; Zavala-Garay et al. 2004; Williams 2005). The model errors may arise from various schemes of physical parameterization (Syu and Neelin 2000), atmospheric noise, or other high-frequency variations, such as westerly wind bursts and the Madden-Julian oscillation (Gebbie et al. 2007; Tang and Yu 2008; Marshall et al. 2009). However, some of these physical processes are omitted in intermediate-complexity models (Zebiak and Cane 1987; McCreary and Anderson 1991), therefore, model errors may exist in these models. Considering the effect of uncertainties in empirical model parameters on ENSO predictability (Mu et al. 2002), Duan and Zhang (2010) and Yu et al. (2012) used an approach of conditional nonlinear optimal perturbation (CNOP) to explore the influence of model parametric errors on ENSO predictability and argued that the parameter errors may have less influence on prediction uncertainties of ENSO. Generally, the model errors consist of the combined effect of uncertainties of model parameters, unrecognized physical processes, sub-grid parameterization, and atmospheric noise etc., and cannot be solely represented by model parametric errors. Furthermore, the effect of model errors on ENSO predictability cannot be only described by parametric errors' effects. It is therefore necessary to explore the effect of all kinds of model errors on ENSO predictability. Actually, we cannot be exact in separation of these kinds of model errors from prediction results; we have to explore the combined effect of these kinds of model errors on ENSO prediction uncertainties.

Roads (1987) superimposed tendency equation with an external forcing term and used it to describe the combined effect of unrecognized physical processes, sub-grid parameterization, atmospheric noise, and so on. Following this idea, Barkmeijer et al. (2003) proposed the forcing singular vector (FSV) concept, which is supposed to be invariant during forecast periods and represents the constant tendency error that has the fastest growth. That is to say, they attempted to reveal the most disturbing tendency errors of the model that tend to yield aggressively large prediction errors. However, the FSV is based on linear theory and cannot depict the effect of nonlinearity, thus not representing the most disturbing tendency error in a nonlinear model. In order to overcome this limitation, Duan and Zhou (2013) extended the FSV to a nonlinear field, i.e., the nonlinear forcing singular vector (NFSV) approach, in which they considered the effect of nonlinearity on tendency errors. The competing aspect of NFSV takes into account the effect of nonlinearity existing in numerical models, and thus is more applicable in describing the most disturbing tendency error in predictability studies associated with model errors.

In this paper, we use the NFSV approach to study the most disturbing tendency error and explore the effect of model errors on prediction uncertainties for ENSO events. We begin by reviewing the NFSV approach in the following section. Then, in Sect. 3, we introduce the intermediate Zebiak-Cane model used in the study. Section 4 explores the NFSVs and FSVs of reference-state El Niño events, reveals the differences between them, and then discusses the mechanism of the evolution of prediction errors caused by the NFSVs. Section 5 investigates the role of nonlinearities in modulating the NFSV-resultant prediction errors. In Sect. 6, we present an interpretation concerning the difference between NFSVs and FSVs. Finally, a summary and a discussion are presented in Sect. 7. 


\section{The nonlinear forcing singular vector}

If we denote the state vector as $\boldsymbol{W}$, the evolution equations for $\boldsymbol{W}$ can be written as

$\left\{\begin{array}{l}\frac{\partial \boldsymbol{W}}{\partial t}=F(\boldsymbol{W}(\boldsymbol{x}, t)), \quad \text { in } \Omega \times[0, \tau] \\ \left.\boldsymbol{W}\right|_{t=0}=\boldsymbol{W}_{0},\end{array}\right.$

where $\boldsymbol{W}(\boldsymbol{x}, t)=\left(w_{1}(\boldsymbol{x}, t), w_{2}(\boldsymbol{x}, t), \ldots, w_{n}(\boldsymbol{x}, t)\right), \boldsymbol{W}_{0}(\boldsymbol{x}, t)$ is the initial state, $(x, t) \in \Omega \times[0, \tau], \Omega$ is a domain in $R^{n}$, $\boldsymbol{x}=\left(x_{1}, x_{2}, \ldots, x_{n}\right), t=0$ is the initial time, and $t=\tau$ with $\tau<+\infty$ is the future time of the evolution of state variables. $F$ is a nonlinear differential operator. We assume that the dynamic system equation and the initial state are known exactly, and the future state can be determined by integrating Eq. (2.1). The solution to Eq. (2.1) for the state vector $\boldsymbol{W}$ at time $\tau$ is given by

$\boldsymbol{W}(\boldsymbol{x}, \tau)=\boldsymbol{M}_{\tau}\left(\boldsymbol{W}_{\mathbf{0}}\right)$.

where $\boldsymbol{M}_{\tau}\left(\boldsymbol{W}_{0}\right)$ is the propagator of the Eq. (2.1). That is to say, the Eqs. (2.1) and (2.2) are in perfect model scenario.

As mentioned above, predictions are generally influenced not only by initial uncertainties, but also model uncertainties. If model uncertainties are assumed to be time-invariant during the forecast period, then the corresponding forecast model, based on the Eq. (2.1), can be described by the following equation:

$\left\{\begin{array}{l}\frac{\partial(\boldsymbol{W}+\boldsymbol{w})}{\partial t}=\boldsymbol{F}(\boldsymbol{W}+\boldsymbol{w})+\boldsymbol{f}(\boldsymbol{x}), \\ \boldsymbol{W}+\left.\boldsymbol{w}\right|_{t=0}=\boldsymbol{W}_{0}+\boldsymbol{w}_{0},\end{array}\right.$

where $\boldsymbol{f}(\boldsymbol{x})$ represents tendency errors, and $\boldsymbol{w}_{0}$ represents initial errors. If we use $\boldsymbol{M}_{\tau}(\boldsymbol{f})$ to denote the propagator of Eq. (2.3), then when $\boldsymbol{f}=0, \boldsymbol{M}_{\tau}(\boldsymbol{f})$ is the same as $\boldsymbol{M}_{\tau}$ in Eq. (2.1). Or say, when $\boldsymbol{f}=0$, Eq. (2.3) represents a perfect model of Eq. (2.1). When $f \neq 0$, ones hope to find the tendency error that causes the largest prediction error at prediction time in predictability studies (Barkmeijer et al. 2003; Duan and Zhou 2013).

Based on Eq. (2.3), Duan and Zhou (2013) defined the NFSV, which represents the constant tendency error that has the largest effect on prediction uncertainties at prediction time. A forcing vector $\boldsymbol{f}_{\delta}$ is the NFSV if and only if

$J_{\delta}\left(\boldsymbol{f}_{\delta}\right)=\max _{\|f\|_{a} \leq \delta} J(f)$,

where

$J(\boldsymbol{f})=\left\|\boldsymbol{M}_{\tau}(\boldsymbol{f})\left(\boldsymbol{W}_{0}\right)-\boldsymbol{M}_{\tau}(0)\left(\boldsymbol{W}_{0}\right)\right\|_{b}$,

and $\|\cdot\|_{a}$ and $\|\cdot\|_{b}$ are measurements of tendency errors $\boldsymbol{f}$ in terms of the norm. The objective function $J$ measures the magnitudes of prediction errors caused by the tendency error $f$.

The NFSV is a natural generalization of the (linear) FSV in a nonlinear field. The so-called FSV was proposed by Barkmeijer et al. (2003) and defined as follows: $\lambda\left(f^{*}\right)=\max _{\boldsymbol{f}} \frac{\left\|\mathbf{M}_{\tau}(\boldsymbol{f})(0)\right\|}{\|\boldsymbol{f}\|}$,

where $\mathrm{M}_{\tau}(\boldsymbol{f})$ is the tangent linear operator of $\boldsymbol{M}_{\tau}(\boldsymbol{f})$ with respect to the reference state $\boldsymbol{W}(\boldsymbol{x}, t)=\boldsymbol{M}_{t}\left(\boldsymbol{W}_{0}\right)$, and the norm $\|\cdot\|$ here is described by the inner product. The vector $f^{*}$ represents the FSV and can be obtained by solving the optimization problem (2.6). The FSV describes the tendency error that has the largest growth rate in the linearized model during the forecast period.

To compute the NFSV, we must numerically solve Eq. (2.4). However, Eq. (2.4) is a maximization problem, and cannot be computed directly. Fortunately, several optimization algorithms are available for calculating minimization problems. Actually, Eq. (2.4) can be transformed into a minimization problem by considering the negative of the objective function. Accordingly, the algorithms for solving minimization problems, such as Spectral Projected Gradient 2 (SPG2; Birgin et al. 2000), Sequential Quadratic Programming (SQP; Powell 1983) and LimitedMemory Broyden-Fletcher-Goldfarb-Shanno (L-BFGS; Liu and Nocedal 1989), can be used to compute the NFSV. In these algorithms, the gradient of the modified objective function [i.e., the negative of the objective function in the Eq. (2.5)] with respect to the external forcing $f$ is required; furthermore, the adjoint of the corresponding models is usually used to obtain the gradient. Duan and Zhou (2013) addressed how to compute the gradient of the objective function with respect to external forcing by using the adjoint and gave the formula of the gradient. With this gradient information, employing the above algorithms can determine the minimum of the modified objective function, i.e., the maxima of the objective function in the Eq. (2.4) along the descending direction of the gradient. In phase space, the point corresponding to the minimum of the modified objective function is the NFSV defined by Eq. (2.4). In the current study, we will use the SPG2 algorithm to obtain the NFSVs of the Zebiak-Cane model with respect to the predetermined model El Niño events.

\section{The Zebiak-Cane model}

The Zebiak-Cane model is a nonlinear anomaly model of intermediate complexity that describes anomalies about a specified seasonally varying background, avoiding the "climate drift" problem. The model is composed of a Gill-type steady-state linear atmospheric model and a reduced-gravity oceanic model, which depict the thermodynamics and atmospheric dynamics of the tropical Pacific with oceanic and atmospheric anomalies (Zebiak and Cane 1987). The atmospheric dynamics are described by the steady-state linear shallow water equations on an equatorial beta plane. 
The circulation is forced by a heating anomaly that depends partially on local heating that is associated with SST anomalies and partially on low-level moisture convergence (parameterized in terms of the surface wind convergence) (Zebiak 1986). In this anomaly, convergence feedback is a nonlinear process because the moisture-related heating occurs only when the total wind field is convergent, which depends not only on the calculated convergence anomaly, but also the specified mean convergence. The important effect of the feedback is to focus the atmospheric response on the SST anomalies in or near the regions of mean convergence, particularly the Intertropical Convergence Zone and the Southern Pacific Convergence Zone. The thermodynamics of this phenomenon are governed by an evolution equation of the SSTA in the tropical Pacific that includes three-dimensional temperature advection by the specified mean currents and the calculated anomalous currents. The assumed surface heat flux anomaly is proportional to the local SST anomaly and constantly adjusts the temperature field toward its climatological mean state, which is specified through observation. In the coupled ZebiakCane model, the atmosphere is first run with the specified monthly mean SST anomalies to simulate monthly mean wind anomalies. Next, the ocean component is enforced by surface wind stress anomalies that are generated from a combination of surface wind anomalies produced by the atmosphere model and the background mean winds.

The Zebiak-Cane model was the first coupled oceanatmosphere model to simulate the observed ENSO interannual variability, and has provided a benchmark in ENSO research over several decades since. In particular, it is one of the few real-time models that successfully predicted the onset of the 91/92 warm ENSO phase. Its successful performance has led to this model being widely used in prediction and predictability studies (Zebiak and Cane 1987; Blumenthal 1991; Xue et al. 1994; Chen et al. 2004; Tang et al. 2008; Mu et al. 2007a; Duan et al. 2009; Yu et al. 2012). There are two types of El Niño events. One type consists of canonical El Niño events, which typically develops from the South American coast and propagates westward across the tropical Pacific (Rasmusson and Carpenter 1982) and is referred to as "EP-El Niño events" in some papers (Kao and Yu 2009; Kug et al. 2009); the other type is a new type El Niño events (often called "CP-El Niño events"), in which warm SST is mainly concentrated in the central Pacific and does not propagate (Ashok et al. 2007). Duan et al. (2013) demonstrated that the Zebiak-Cane model tends to be well in simulating the EP-El Niño events but bad in depicting the CP-El Niño events. Therefore, the Zebiak-Cane model may describe the essential physics of EP-El Niño and can be regarded as a tool for investigating the effect of superimposed tendency errors on the predictability of EP-El Niño events. That is to say, the results derived from the
Zebiak-Cane model are generally for EP-El Niño events in a perfect model scenario.

\section{The NFSVs of the Zebiak-Cane model with respect to predetermined El Niño events}

Perfect model predictability experiments have been widely used in predictability studies, in which numerical models are assumed to be perfect and perturbations are superimposed on initial conditions or model parameters or tendency equations to explore the effect of initial errors or model errors on predictability (Lorenz 1996; Moore and Kleeman 1996; Barkmeijer et al. 2003; Mu and Zhang 2006; Duan et al. 2009; Duan and Zhou 2013, etc.). In this paper, we assume the Zebiak-Cane model to be perfect and conduct the predictability experiments in a perfect model scenario (see Sect. 3), in which a tendency error is superimposed to the Zebiak-Cane model for describing an imperfect forecast model [see Eq. (4.1)] and explore which feature displays the most disturbing tendency error associated with El Niño predictions, which, as mentioned in Sect. 3, is for EPEl Niño events. For simplicity, we still use the terminology "El Niño" to describe the results in the context.

$$
\begin{aligned}
\partial T / \partial t= & -U \cdot \nabla(\bar{T}+T)-\bar{U} \cdot \nabla T-\left[M\left(\bar{w}_{s}+w_{s}\right)-M\left(\bar{w}_{s}\right)\right] \times \bar{T}_{z} \\
& -M\left(\bar{w}_{s}+w_{s}\right) T_{z}-\alpha_{s} T+\boldsymbol{f}(\boldsymbol{x}, \boldsymbol{y}) .
\end{aligned}
$$

where $T, U, w_{s}$, and $\_\bar{w}_{s}$, denote anomalies of mixed layer temperature (or SST), horizontal surface velocity (a vector), the upwelling at the mixed layer base, and the mean upwelling. The coefficient $\alpha_{s}$ is a nondimensional parameter that represents the Newtonian cooling coefficient for SSTA. The function $M(x)$ is defined by

$M(x)= \begin{cases}0, & x \leq 0 ; \\ x, & x>0 .\end{cases}$

It accounts for the fact that surface temperature is affected by vertical advection only in the presence of upwelling. The anomalous vertical temperature gradient, $T_{z}$, is defined by

$T_{z}=\frac{T-T_{e}}{H_{1}}$

where $H_{1}$ is the surface layer thickness, and Te measures the temperature anomalies entrained into the surface layer.

Tropical Pacific SSTAs comprise one of the main components of the interannual variations of the coupled system in the core region of the ENSO. To utilize the Zebiak-Cane model to study the effect of tendency errors on the prediction uncertainties for ENSO, we consider superimposing a tendency error to the SSTA equation that describes the 
interannual variations of ENSO and explore the NFSVs of the Zebiak-Cane model with respect to some predetermined reference-state El Niño events [(see Eq. (4.1)]. We define the objective function associated with the NFSV [i.e., the vector $\boldsymbol{f}_{\delta}$ in Eq. (4.2)] as follows:

$$
J\left(\boldsymbol{f}_{\delta}\right)=\max _{\|\boldsymbol{f}\|_{b} \leq \delta}\left\|T^{\prime}(\tau)\right\|_{a},
$$

where the norm $\left\|T^{\prime}(\tau)\right\|_{a}=\sqrt{\sum_{i, j}\left(T_{i, j}^{\prime}(\tau)\right)^{2}}$ is used to measure the amplitude of the prediction error caused by the tendency error $\boldsymbol{f}$ and the norm $\|\boldsymbol{f}\|_{b}=\sqrt{\sum_{i, j}\left(f_{i, j}\right)^{2}}$ is adopted to constrain the magnitude of tendency error $f$. Here, $T^{\prime}(\tau)$ represents the evolution of the error of predictions for the SSTA component caused by tendency error at future time $\tau$, and is obtained by subtracting the SSTA of the reference-state El Niño events from the predicted SSTA generated by the Zebiak-Cane model (with the tendency error $f$ ) at prediction time $\tau$. $T_{i, j}^{\prime}$ represent the prediction error of the SSTA at different grid points and $(i, j)$ is the grid point in the domain of the tropical Pacific with latitude and longitude, respectively, from $129.375^{\circ} \mathrm{E}$ to $84.375^{\circ} \mathrm{W}$ by $5.625^{\circ}$ and from $19^{\circ} \mathrm{S}$ and $19^{\circ} \mathrm{N}$ by $2^{\circ}$. The NFSV is computed for the time period from the start time to the future prediction time [i.e., the time interval $(0, \tau)$ ], which is also called the optimization time period of determining the NFSV.

We first determine the reference-state El Niño events to be predicted. Mu et al. (2003) and Duan et al. (2012) demonstrated that the CNOP-type initial anomalies are most likely to evolve into El Niño events and act as the optimal precursory disturbance for El Niño events. Furthermore, the CNOP-resultant El Niño events sufficiently consider the effect of nonlinearity. In this study, we choose the CNOP-resultant El Niño events as reference states to be predicted, which may be convenient for revealing the effect of nonlinearity on the prediction errors caused by the tendency errors. The CNOP-type initial anomalies of the Zebiak-Cane model are initialized in January and determined by constraint conditions with magnitudes of $\sigma=0.4,0.6,0.8$ and 1.0 (see the "Appendix"). These CNOP-initial anomalies tend to exhibit a zonal dipolar SSTA pattern with positive anomalies in the equatorial eastern Pacific and negative anomalies in the equatorial central-western Pacific, and a thermocline depth anomaly pattern with positive anomalies along the equator (Fig. 1). Such patterns are favorable for a strong equatorial eastwest thermal contrast and a deepening thermocline depth along the equator, which then easily induce a strong equatorial westerly anomaly and initial warm subsurface water, thereby causing the temperature of the upwelled water to become warmer and finally yielding El Niño events [the details can be referred to in Duan et al. (2012)]. Figure 2 plots the Niño-3 index of the El Niño events induced by the CNOP-type initial anomalies in Fig. 1. It can be seen that the CNOP-type initial anomalies evolve into El Niño events with different intensities; specifically, the larger the magnitudes (i.e., values of $\sigma$ ) of CNOP-type initial anomalies, the stronger the corresponding El Niño events. The four El Niño events shown in Fig. 2 often present their peak phases in boreal winter and persist for roughly more than 20 months. The properties of these model El Niño events are very similar to the observed El Niño events. It is therefore reasonable for us to use these model El Niño events as reference states to obtain the NFSV-type tendency errors.

In this context, we use Year $(0)$ to denote the year when El Niño attains a peak value, and Year $(-1)$ and Year (1) to signify the year before and after Year (0), respectively. If the reference-state El Niño events are assumed to be predicted with 1 year lead time by using the ZebiakCane model with tendency errors $f$, then the optimization time period of determining the NFSVs is 12 months. In the numerical experiments, the El Niño predictions are first made with a start month of January (0) [i.e., January in Year (0)], April (0), and July (0). Then, the NFSVs are determined with these start months and optimization time period of 12 months. These NFSVs are mainly associated with the predictions for the growth phase of El Niño; and for convenience, we hereafter refer to these predictions as growth-phase predictions and the NFSVs as the ones of the growth-phase predictions, which represent the constant tendency errors that have the largest effect on the uncertainties of the growth-phase predictions. Subsequently, we perform further numerical experiments for El Niño predictions with a start month of January (1) [i.e., January in Year (1)], April (1), and July (1) and a lead time 12 months. These three predictions are the ones crossing through the decaying phase of El Niño and are therefore referred to hereafter as decaying-phase predictions; and the corresponding NFSVs, with the optimization time period being 12 months, are described as the ones of the decaying-phase predictions for El Niño events.

For the NFSVs, we choose for the experiments the constraint bounds $\delta$ [see Eq. (3.1)] as 0.8, 1.0, and 1.2, to constrain the magnitudes of tendency errors $f$ of SSTA. As a result, we will have 18 predictions for each El Niño event, as shown in Fig. 1, a total of 72 predictions (36 growthphase predictions and 36 decaying-phase predictions) for the four El Niño events, and are then required to compute the NFSVs of 72 predictions. Computations show that, for each value of $\delta$, regardless of start month, there exists one NFSV for each prediction. Then, we obtain the 72 NFSVs. The magnitudes of all these NFSVs in terms of the chosen norm are always equal to the values of the constraint bounds $\delta$. That is, all of these NFSVs locate on the boundary of the corresponding constraint condition $\|\boldsymbol{f}\|_{b} \leq \delta$. These NFSVs consist of the SSTA component and, for the 


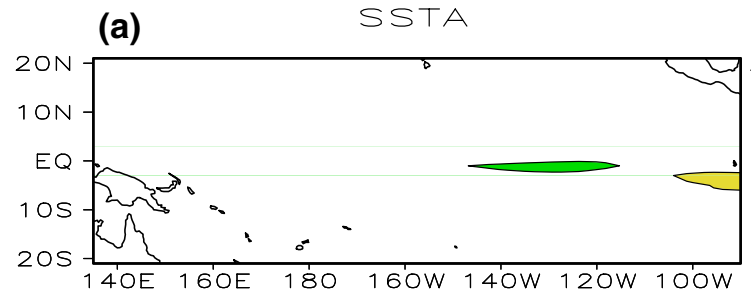

(b)

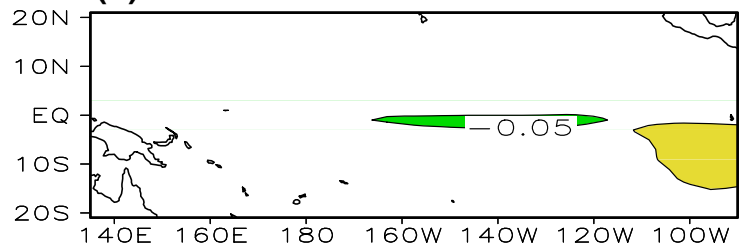

(c)

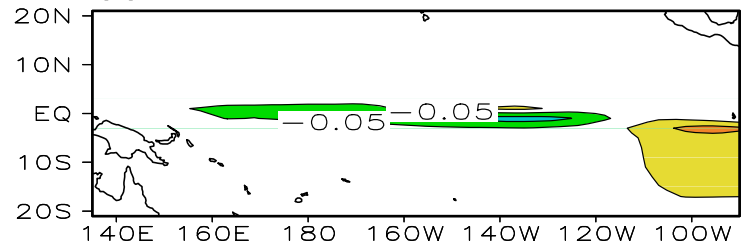

(d)

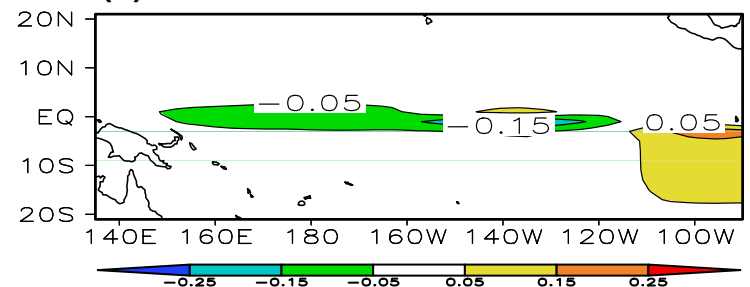

Fig. 1 The SST and thermocline depth anomalies of the CNOP-type initial anomalies. a The initial anomalies with magnitude of $\sigma=0.4$; $\mathbf{b}, \mathbf{c}$, and $\mathbf{d}$ as in (a) but with magnitudes of $\sigma=0.6, \sigma=0.8$, and

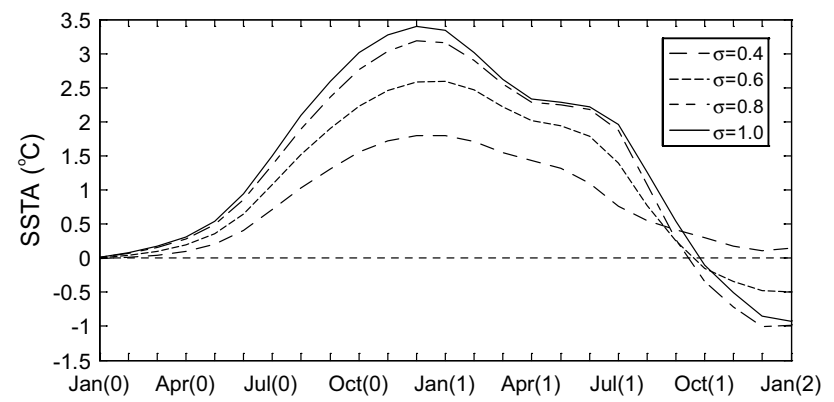

Fig. 2 Niño-3 indices of the El Niño events caused by the CNOPtype initial anomalies shown in Fig. 1

reference-state El Niño events with different intensities, often exhibit similar large-scale zonal dipolar patterns for a particular initial time. More specifically, the NFSVs of the growth-phase predictions present a zonal dipolar pattern with positive anomalies in the equatorial central-western
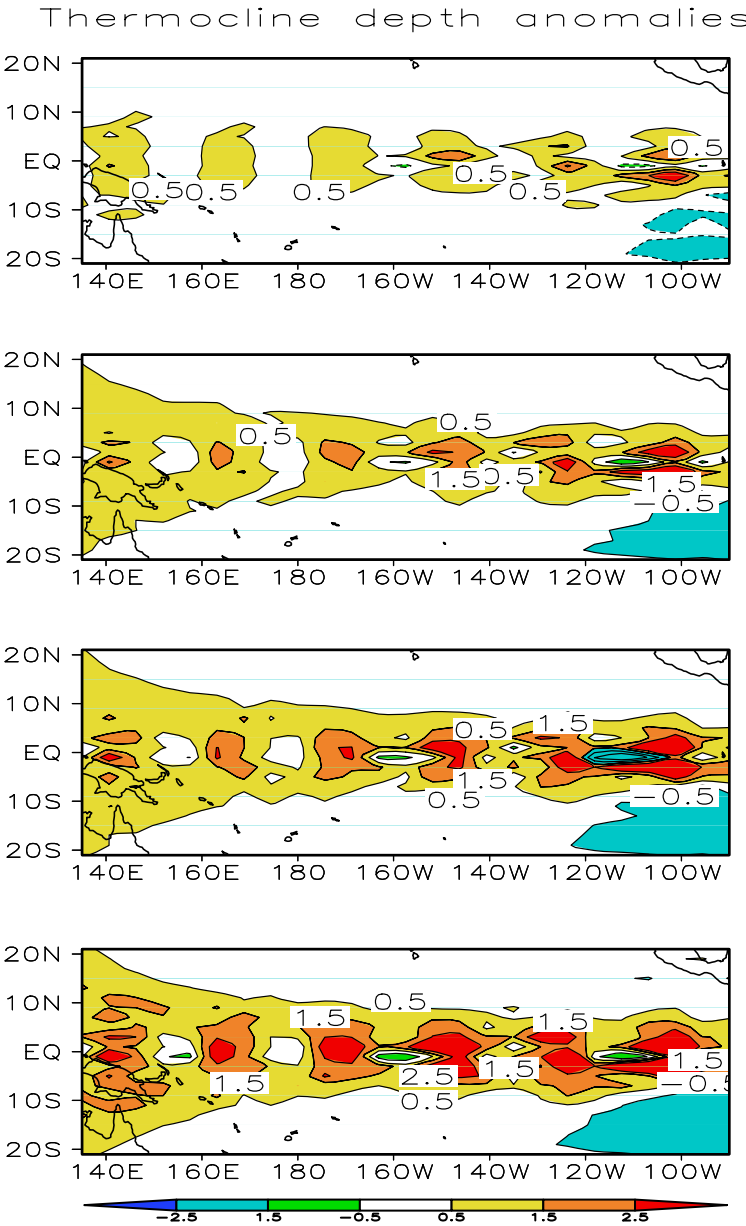

$\sigma=1.0$, respectively. These initial anomalies are initialized in January, with optimization periods of 12 months

Pacific and negative anomalies in the equatorial eastern Pacific (hereafter denoted by "NFSV1"). However, those of the decaying-phase predictions have signs almost opposite to the former (hereafter denoted by "NFSV2").

Figure 3 plots the NFSV-type tendency errors of the growth-phase predictions (for the El Niño event with $\sigma=1.0$ ) with the start month being January (0) and the constraint bounds of tendency errors being $\delta=0.8,1.0,1.2$, respectively. It can be seen that, although all these tendency errors tend to present the NFSV1 pattern, there exist differences among them for different constraint bounds. In particular, the NFSV1 with large magnitudes tend to extend their positive anomalies much farther westward. In any case, from the pattern of the NFSV1, it is inferred that the NFSV1 tendency errors tend to enhance the equatorial eastern cooling-western warming thermal contrast and induce strong easterly anomalies, which are superimposed on the SSTA equation of the Zebiak-Cane model and persist to force the tropical Pacific SSTA. Subsequently, a persistent 
(a)

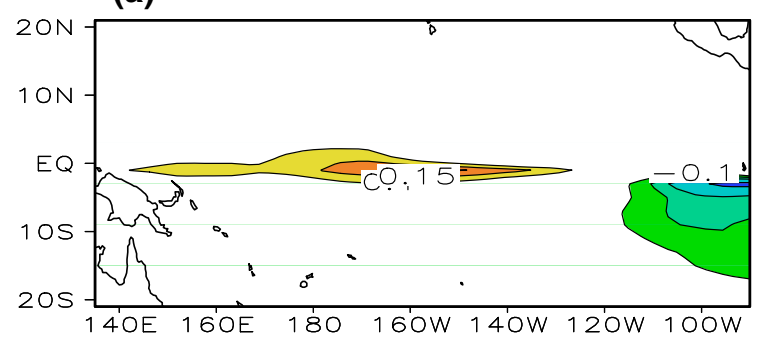

(b)

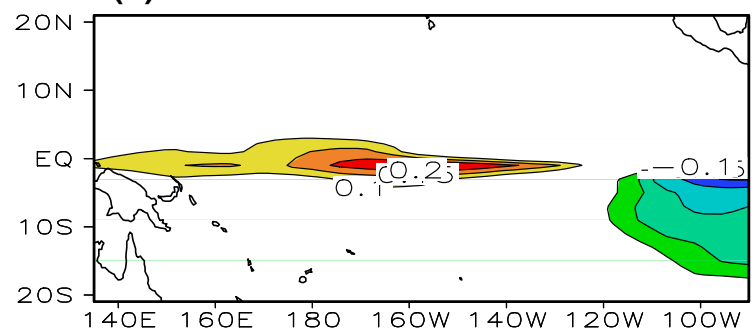

(c)

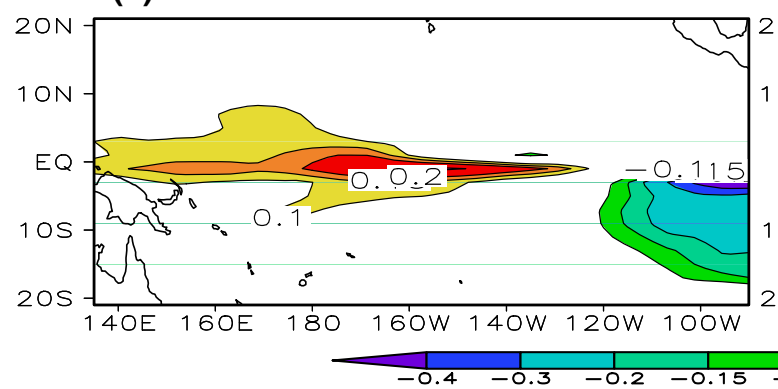

Fig. 3 SSTA component of FSV1 and NFSV1 of the El Niño event (induced by the CNOP-type initial anomaly with magnitude of $\sigma=1.0$ ). These NFSVs and FSVs are, respectively, of magnitudes of

anomalous upwelling may occur in the equatorial eastern Pacific, and the upwelled sub-surface cold water can suppress the warming of El Niño events, finally weakening the reference-state El Niño events and yielding a negative prediction error of the Niño-3 SSTA for the El Niño events.

To confirm this inference, we investigate the prediction errors of El Niño events caused by the NFSV1 tendency errors. With the initial anomalies of the reference-state El Niño events as the initial values, we integrate the ZebiakCane model with the NFSV1 tendency error for one model year and obtain the predictions for El Niño events. Subtracting the reference-state El Niño events from their predictions provide the prediction errors caused by the NFSV1 tendency errors with lead times of three, six, nine, and 12 months and the corresponding thermocline depth and wind stress anomalies. We determine that, no matter which El Niño event is predicted, and whatever the initial times are, the NFSV1 tendency errors with $\delta=0.8,1.0,1.2$ always cause significantly negative prediction errors of the Niño-3 SSTA. Furthermore, the prediction errors caused $\mathbf{a} \delta=0.8, \mathbf{b} \delta=1.0$, and $\mathbf{c} \delta=1.2$, and are calculated with the start month of January (0) and the optimization period being 12 months

by the NFSV1 tend to behave as a La Niña-like evolving mode. Figure 4 presents the SST, thermocline depth and wind stress anomalies of the prediction errors (of the El Niño event with $\sigma=1.0$ ) caused by the NFSV1 of $\delta=1.0$ with the start month January (0). The results demonstrate that the NFSV1 tendency errors induce anomalous easterly forcing and decrease the thermocline depth in the eastern Pacific, which finally suppresses the evolution of El Niño events and causes a negative prediction error for Niño-3 SSTA of El Niño events (see Table 1).

For the NFSV2 tendency errors, we plot in Fig. 5 the cases for the El Niño event with $\sigma=1.0$, with January (1) in the decaying phase of the El Niño as the start month of predictions, and $\delta=0.8,1.0,1.2$ as the constraint bounds of tendency errors' magnitudes, respectively. Indeed, the NFSV2 are almost opposite to the NFSV1 and have the pattern with negative anomalies in the equatorial central-western Pacific and positive anomalies in the equatorial eastern Pacific. Furthermore, they behave as an El Niño-like evolving mode (Fig. 6), which is contrary to the La Niña-like 
SSTA

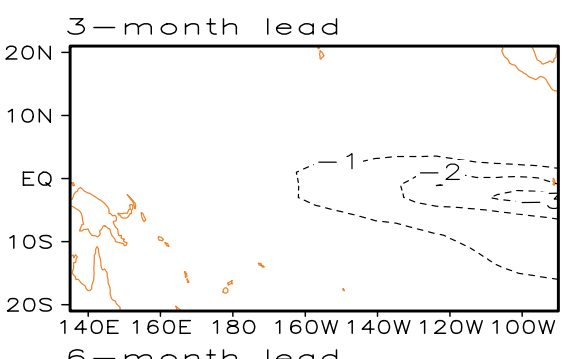

6 - month lead

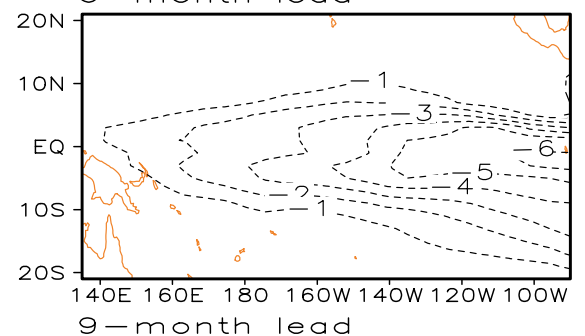

9 - month lead

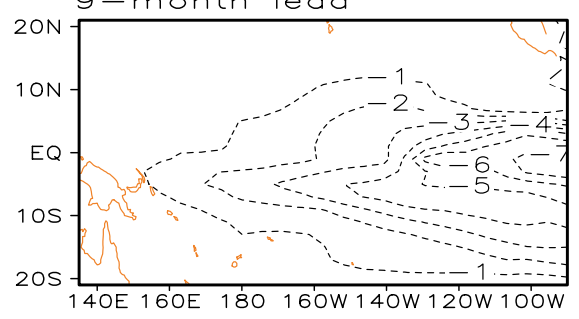

$12-$ month lead

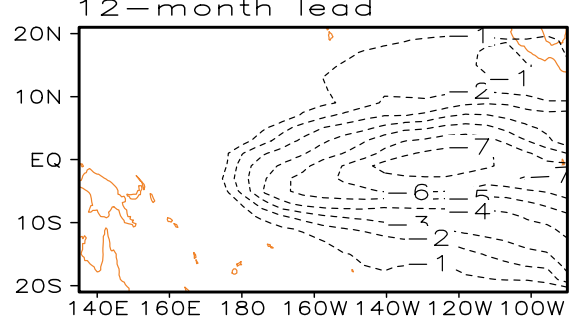

Thermocline depth anomalies Zonal wind anomalies
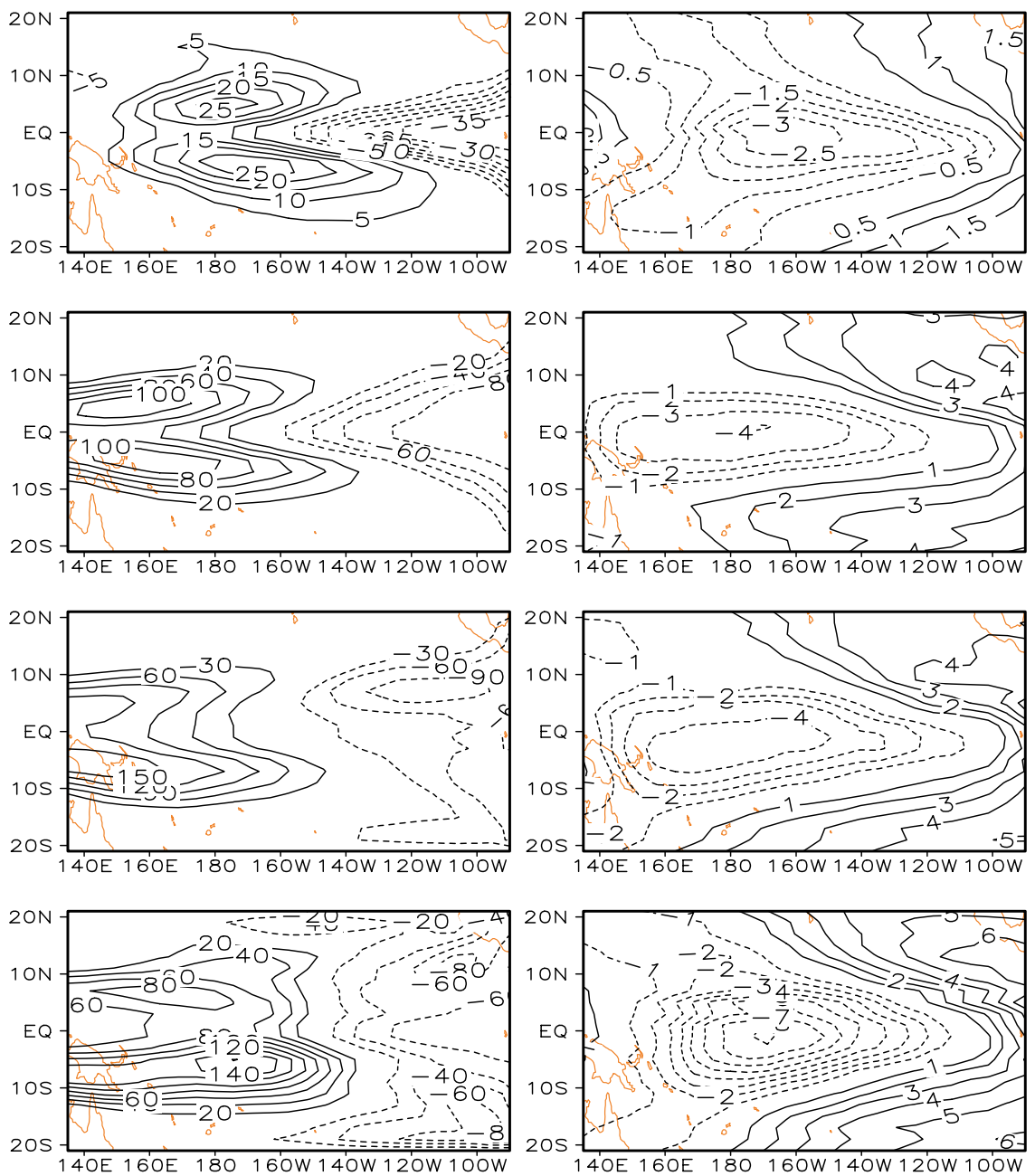

Fig. 4 The evolution patterns of the prediction errors caused by the NFSV1 shown in Fig. 3, but only for that with magnitude of $\delta=1.0$. The left, middle, and right columns describe the SSTA, thermocline depth anomaly, and the corresponding zonal wind anomaly, respectively

evolving mode of the NFSV1. That is to say, the NFSV2 cause a positive prediction error of the Niño-3 SSTA for the reference-state El Niño events (see Table 1).

The NFSV is a nonlinear extension of the FSV. For comparison, we compute the FSVs of the growth-phase and decaying-phase predictions for the reference-state El Niño events by using the linearized Zebiak-Cane model. Note that if $f_{L}$ is an FSV, the vectors $c f_{L}$ ( $c$ is a real number) are also FSVs with the same growth rate as the FSV $f_{L}$. The NFSVs differ from the FSVs. If $f_{\delta}$ is an NFSV, $c f_{\delta}$ may not be an NFSV because of the effect of the nonlinearities. Therefore, a given NFSV should be compared with the FSV with the same magnitude. For this reason, we define a scaled FSV as follows:

$\hat{f}_{L}=(-1)^{N} \frac{\left\|f_{\delta}\right\|}{\left\|f_{L}\right\|} f_{L}, N=1,2$
Thus, $\left\|\hat{f}_{L}\right\|=\left\|f_{\delta}\right\|$. If the sign of the FSV $f_{L}$ is opposite to (or the same as) that of the NFSV $f_{\delta}$, the "N" in Eq. (4.2) is equal to 1 (or 2). Then, the NFSV $f_{\delta}$ and the scaled FSV $\hat{f}_{L}$ have the same signs and magnitudes. The following comparison between the NFSV and the FSV is conducted under this condition.

Results demonstrate that the FSVs also exhibit a largescale zonal dipolar pattern similar to the NFSVs. However, for different values of $\delta$, the FSVs respectively present their western and eastern poles in almost common locations; while the NFSVs, particularly for large values of $\delta$, tend to extend their western poles much farther westward and cover a much broader spatial region. If we make the FSVs of growth-phase predictions have signs as in the NFSV1 and those of the decaying-phase predictions have signs as in the NFSV2, then the FSVs can also be classified into two 
Table 1 Prediction errors for Niño-3 SSTA of an El Niño event caused by the NFSVs and FSVs (for $\delta=0.8,1.0$, and 1.2), where the El Niño is induced by the CNOP-type initial anomaly with $\sigma=1.0$

\begin{tabular}{lccc}
\hline$\delta$ & 0.8 & 1.0 & 1.2 \\
Start month Jan (0) & & & \\
FSV & -3.64 & -5.15 & -6.34 \\
NFSV & -3.68 & -5.81 & -7.07 \\
Start month Apr (0) & & & \\
$\quad$ FSV & -3.01 & -5.38 & -5.69 \\
NFSV & -3.21 & -5.82 & -6.98 \\
Start month Jul (0) & & & \\
FSV & -3.57 & -5.86 & -5.02 \\
NFSV & -3.65 & -5.96 & -6.09 \\
Start month Jan (1) & & & \\
FSV & 4.13 & 4.40 & 4.57 \\
NFSV & 4.49 & 5.02 & 6.69 \\
Start month Apr (1) & & & \\
FSV & 3.30 & 4.28 & 4.58 \\
NFSV & 3.95 & 5.52 & 5.48 \\
Start month Jul (1) & & & \\
FSV & 3.55 & 4.03 & 4.63 \\
NFSV & 3.68 & 5.81 & 6.24 \\
\hline
\end{tabular}

types: FSV1 and FSV2. As examples, we plot in Figs. 3 and 5 the corresponding FSV1 with the start month being January (0) and the FSV2 with the start month being January (1). The results show that the FSV1, as the NFSV1 do, often cause negative prediction errors of Niño-3 SSTA of El Niño events; while the FSV2, like the NFSV2, yield positive prediction errors for the Niño-3 SSTA. Despite this, the FSVs often cause a much smaller prediction error than the corresponding NFSVs (see Table 1). It is therefore inferred that the tendency errors of the NFSV structures will clearly be more likely to cause a much larger prediction error than the tendency errors of the FSVs. Therefore, the tendency errors of the NFSV structure may be more applicable than the FSV in describing the most disturbing tendency errors that have the largest effect on the prediction errors.

\section{The role of nonlinearity in modulating the NFSV-resultant prediction errors}

NFSVs are derived from a nonlinear model, whereas FSVs originate from the linearized version of the nonlinear model. It is clear that the differences between the patterns of the NFSVs, particularly for those with large magnitudes, and those of the corresponding FSVs, result from the effects of the nonlinearities, which finally causes the difference in their resultant prediction errors. This outcome indicates that the prediction errors caused by the NFSVs with larger magnitudes are more significantly influenced by nonlinearities. But how do nonlinearities influence the NFSV-resultant prediction errors?

To address the behavior of nonlinearities modulating NFSV-resultant prediction errors, we choose magnitudes of tendency errors that are favorable for revealing the effect of nonlinearities. As shown above, choosing the large values of $\delta$ contributes to testing the effect of nonlinearities on prediction errors caused by the tendency errors. Specifically, we choose the NFSVs with the constraint $\delta=1.0$.

We superimpose the SSTA equation of the ZebiakCane model with FSV-type and NFSV-type tendency errors and integrate the perturbed model for 12 months, obtaining the predictions for the reference-state El Niño events. By subtracting the reference-state El Niño events from their predictions, the NFSV- and FSV-resultant prediction errors can be obtained. Similarly, we superimpose the FSV- and NFSV-tendency errors to the linearized ZebiakCane model with respect to the reference-state El Niño events and integrate it for 12 months, obtaining the prediction errors caused by the FSV and NFSV in the linearized Zebiak-Cane model. By comparison, we identify the effect of nonlinearity on prediction errors caused by the tendency errors. For El Niño events with different intensities, we obtain similar results. Next, we take the El Niño event induced by the CNOP-type initial anomaly with $\sigma=1.0$ (see the Appendix) as an example to describe the results.

The SSTA component of the prediction errors caused by the NFSV1 and FSV1 tendency errors with the start month being January (0) is plotted in Fig. 7. It can be seen that the prediction errors induced by the FSV1 tendency errors in the linearized Zebiak-Cane model are significantly larger than those caused by those in the nonlinear model. This indicates that the model's nonlinearities suppress the evolution of prediction errors caused by the FSV1 tendency errors, and also that the nonlinearities have a damping effect on the growth of the prediction errors associated with the growth-phase predictions for El Niño events. However, when we focus on the prediction errors caused by the FSV1 and NFSV1 tendency errors in the nonlinear model, we can see that the prediction errors caused by the NFSV1 are certainly larger than those caused by the FSV1 in the nonlinear model. It is obvious that the structure of the NFSV1 tendency errors is particularly favorable for reducing the suppression effect of the nonlinearities on the growth of prediction errors of the growth-phase predictions for El Niño.

For the FSV2 and NFSV2 tendency errors, they, as demonstrated above, often cause positive prediction errors of the Niño-3 SSTA for the decaying-phase predictions of El Niño events. Although both FSV2 and NFSV2 have dynamic behaviors almost opposite to those of FSV1 and NFSV1, they also usually yield much larger prediction 
(a)

FSV

NFSV
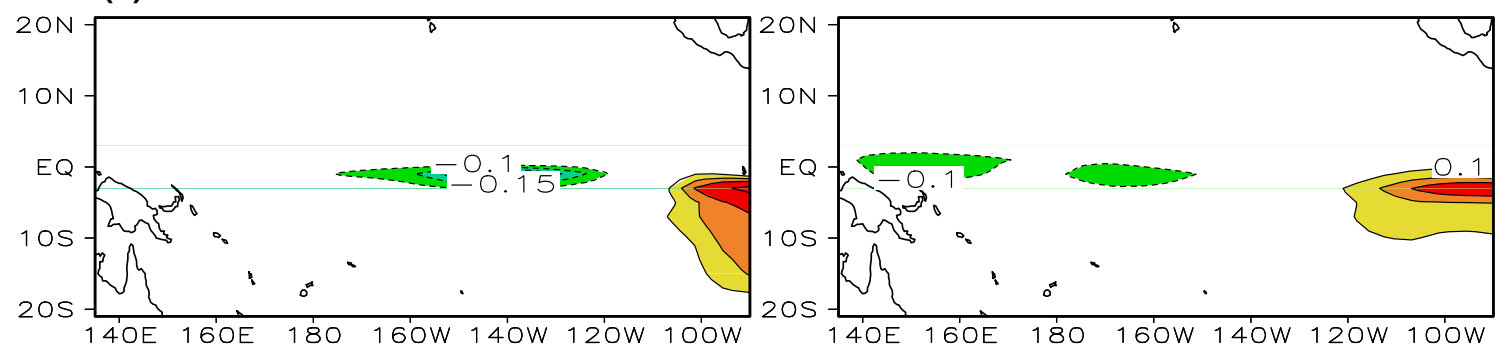

(b)
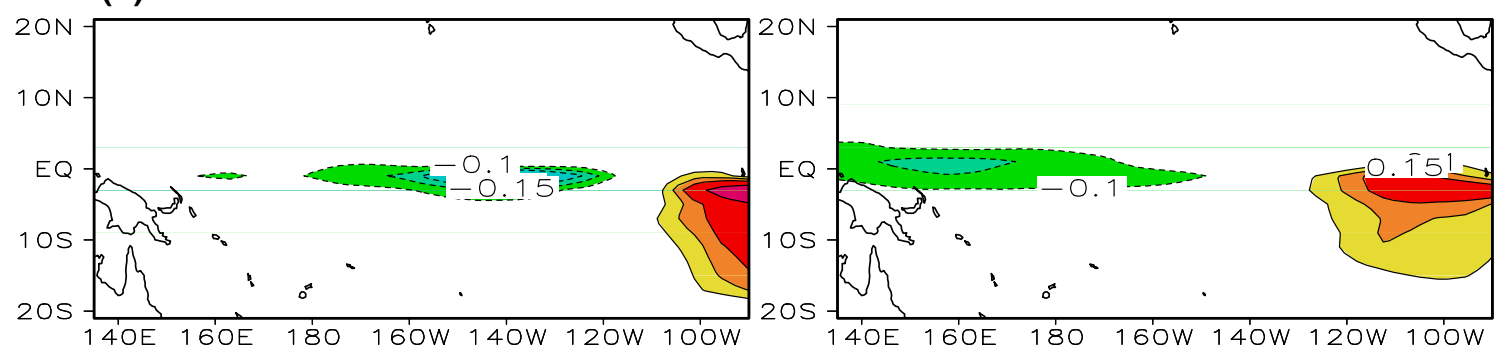

(c)

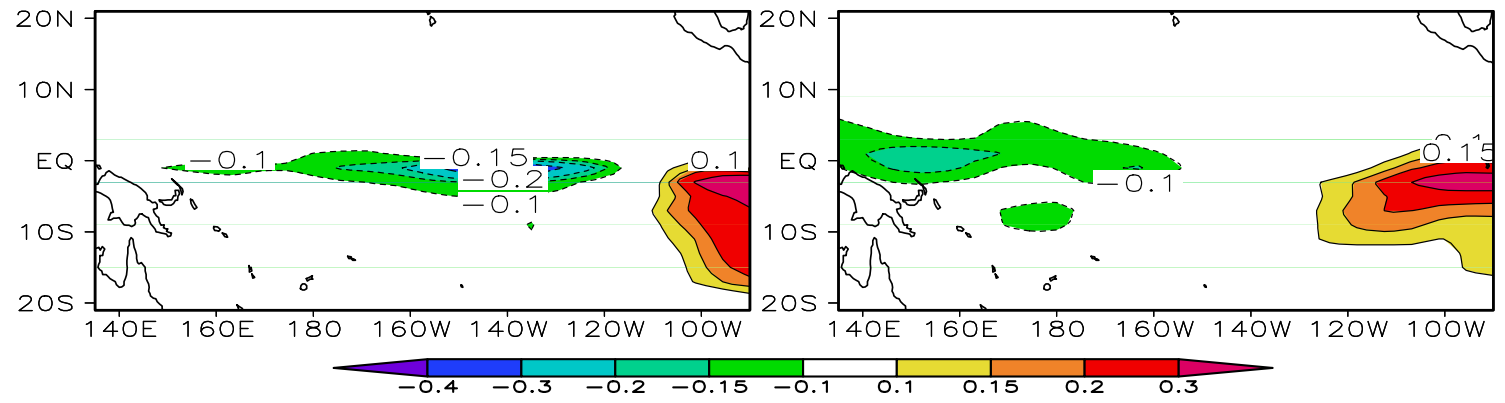

Fig. 5 As in Fig. 3, but for FSV2 and NFSV2

errors in the linearized Zebiak-Cane model than in the nonlinear model (see Fig. 8), and the nonlinearities have a suppressing effect on the growth of prediction errors. Furthermore, considering the NFSV2 certainly yield much larger prediction errors than the FSV2 in the nonlinear model, we also conclude that the particular structure of NFSV2 is favorable for reducing the suppressing effect of nonlinearities on the growth of prediction errors associated with the decaying-phase predictions for El Niño.

We have showed that the FSVs, compared to the NFSVs, tend to yield much larger prediction errors in the linearized model, but much smaller prediction errors in the nonlinear Zebiak-Cane model. This does not mean that we, since the FSV causes much small prediction errors in the nonlinear model, should adopt here the results of FSV. In fact, we attempt to reveal the most disturbing tendency error of the Zebiak-Cane model associated with El Niño predictability. Therefore, we should explore the tendency error that causes the largest prediction error. The NFSVs yield much larger prediction errors than the FSVs in the nonlinear
Zebiak-Cane model and are then more applicable for representing the most disturbing tendency error. Although the FSVs cause larger prediction errors than the NFSVs in the linearized model, the linearized model is an approximation to the nonlinear Zebiak-Cane model and then the resultant FSVs cannot represent the most disturbing tendency error of the nonlinear Zebiak-Cane model.

\section{Interpretation}

The above reported results demonstrated that the nonlinearities have a damping effect on the growth of the prediction errors caused by the FSV1 and FSV2. That is to say, the FSVs result in much smaller prediction errors in the nonlinear Zebiak-Cane model than in its linearized version and nonlinearities suppress the growth of prediction errors caused by the FSVs. The nonlinearities in the Zebiak-Cane model are mainly from the nonlinearities in perturbation temperature advection, wind stress anomalies 


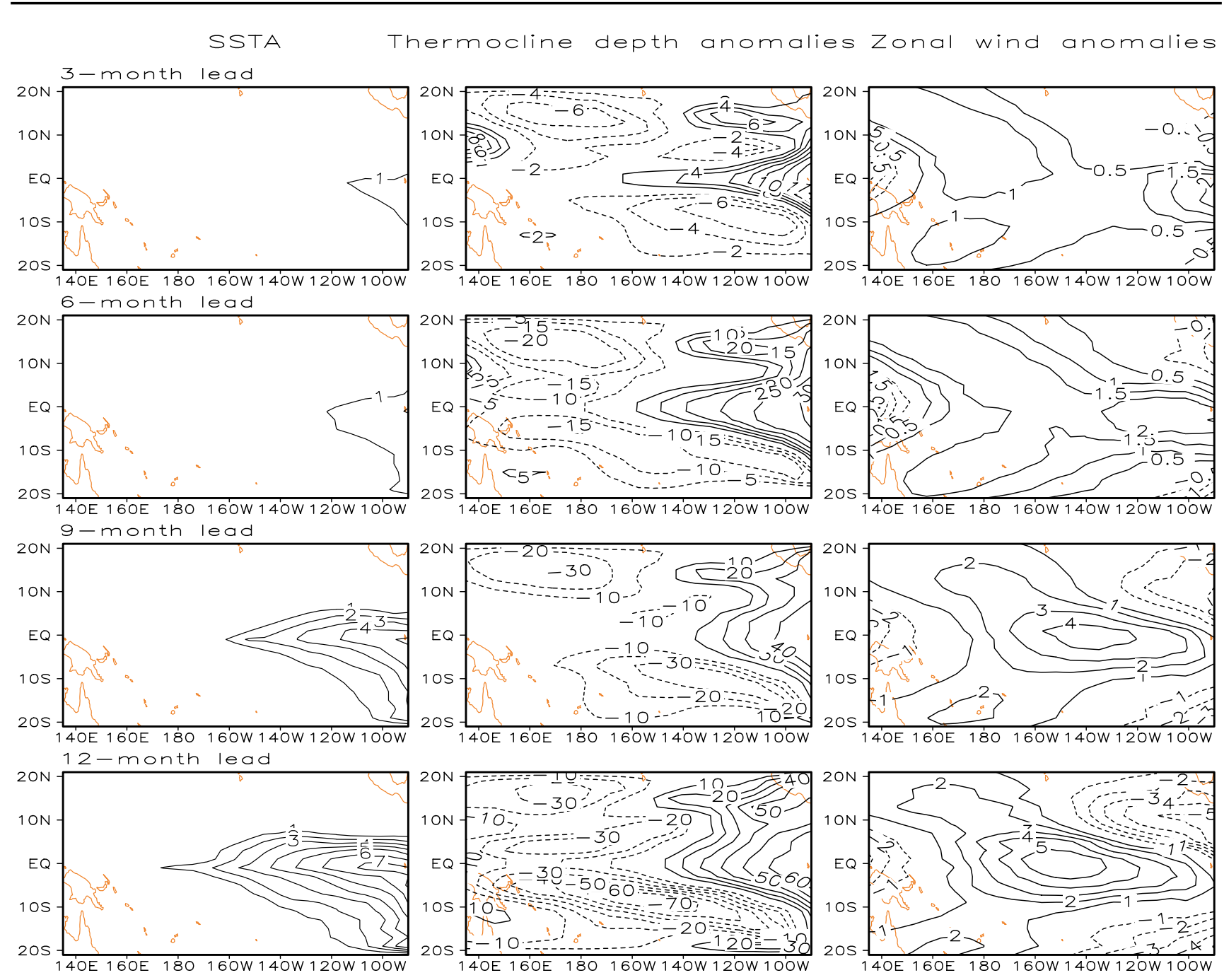

Fig. 6 As in Fig. 4, but for NFSV2

formulation, and sub-surface temperature parameterization. Duan et al. (2008) demonstrated that the perturbation temperature advection always shows positive values in the equatorial eastern Pacific during either an El Niño or a La Niña event, enhancing the former and suppressing the latter. Meanwhile, the nonlinearity in wind stress anomalies suppresses El Niño and enhances La Niña, and that associated with the sub-surface temperature parameterization suppresses both El Niño and La Niña. In this paper, we have shown that the prediction errors caused by the FSV1 exhibit a La Niña evolving mode and those caused by the FSV2 tend to present an El Niño evolving mode. It is therefore inferred that the nonlinearity in the perturbation temperature advection may suppress the prediction errors caused by the FSV1 but enhance those caused by the FSV2. Meanwhile, the nonlinearity in wind stress anomalies may increase the prediction errors caused by the FSV1 and reduce those caused by the FSV2, and that in the sub-surface temperature parameterization suppresses the prediction errors caused by both FSV1 and FSV2. We have demonstrated that the combined effect of three kinds of nonlinearities is to suppress the prediction errors caused by the FSV1 and FSV2. It is inferred that, for the prediction errors caused by the FSV1, the suppression effects of both perturbation temperature advection and sub-surface temperature parameterization may be larger than the enhancement effect of the wind stress anomalies, finally causing the combined effect of three kinds of nonlinearities to suppress the prediction errors. Meanwhile, for those caused by the FSV2, the nonlinear enhancement effect of perturbation temperature advection may be smaller than the suppression effect of nonlinearities in both sub-surface temperature parameterization and wind stress anomalies, which then also results in the combined effect of three kinds of nonlinearities to suppress the growth of prediction errors. 

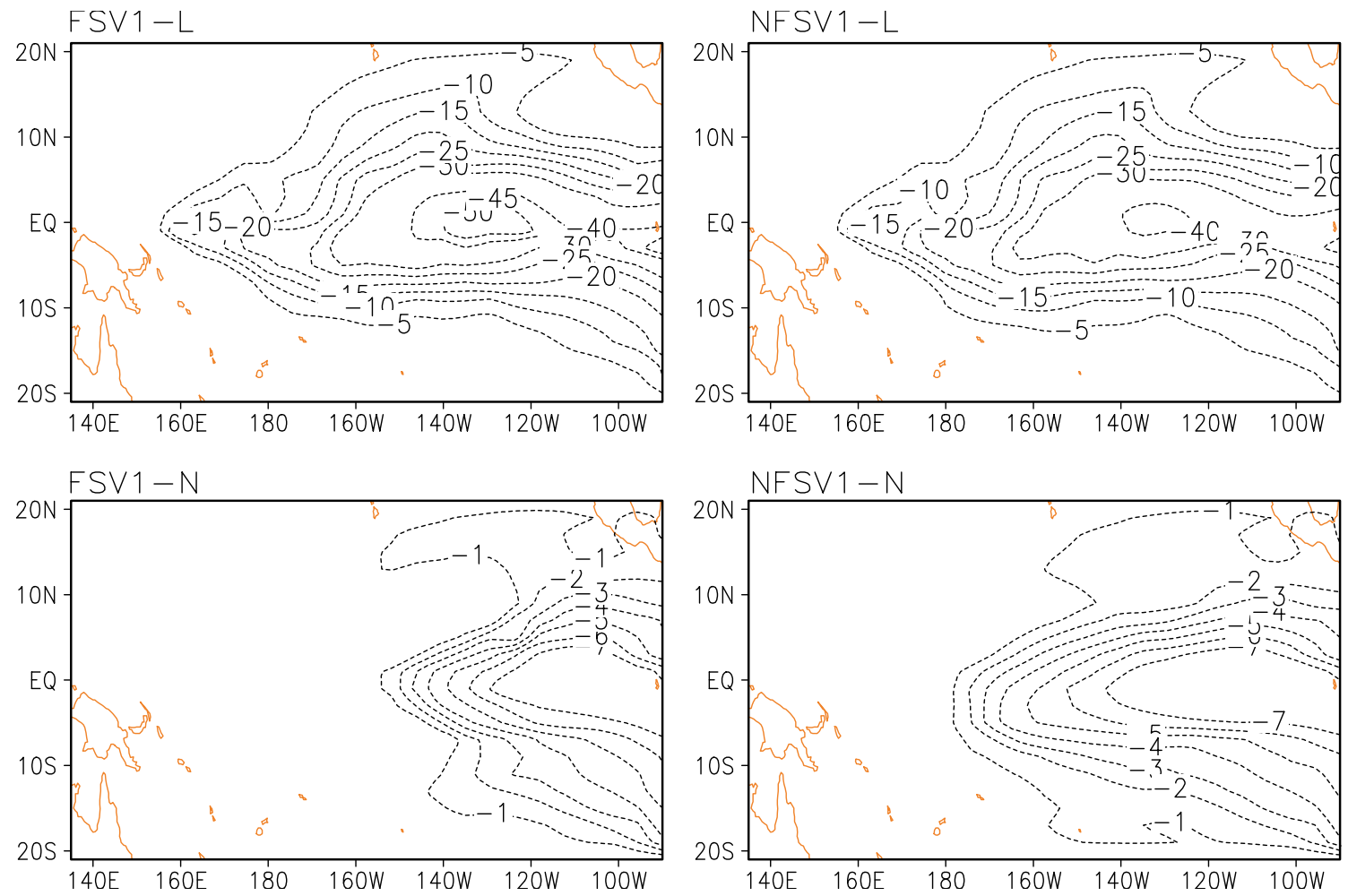

Fig. 7 The SSTA component of prediction errors caused by the NFSV1 and FSV1 in the nonlinear Zebiak-Cane model (denoted by "NFSV1-N" and "FSV1-N", respectively) and those caused by the NFSV1 and FSV1 in the linearized Zebiak-Cane model (denoted by

"NFSV1-L" and "FSV1-L", respectively). The prediction period is 12 months, with the start month being January (0), and the magnitude of NFSV and FSV is $\delta=1.0$

We have also shown that the NFSV1 and NFSV2 favor reducing the damping effect of nonlinearities on prediction errors caused by the FSVs. The NFSV1 and NFSV2, particularly for those of large magnitudes, compared to the FSV1 and FSV2, tend to present their two western poles much farther western. In fact, the NFSV1 (and NFSV2) patterns can be understood as the composite of the FSV1 (and FSV2) patterns and an additional SSTA tendency error of a zonal dipolar structure with positive (negative) anomalies in the equatorial western Pacific and negative (positive) anomalies in the equatorial eastern Pacific (see Fig. 9). The additional SSTA tendency errors, having patterns similar to the NFSV1 (NFSV2), easily induce anomalous easterlies (westerlies) along the equatorial Pacific, which are superimposed on the FSV1 (FSV2)-induced anomalous easterlies (westerlies) in the equatorial eastern Pacific and induce much stronger upwelling (downwelling) to occur in a much broader region in the equatorial central-eastern Pacific, ultimately yielding much larger but negative (positive) prediction errors of Niño-3 SSTA for El Niño events. It is clear that the role of the structure of NFSVs in yielding prediction errors is to increase the prediction errors, while that of nonlinearities is to suppress the prediction errors. Therefore, when the effect of the NFSVs' structure

on prediction errors is superimposed on that of nonlinearities in the Zebiak-Cane model, the particular structure of NFSVs tends to reduce the damping effect of nonlinearities on prediction errors caused by the FSVs.

From the above analysis, it is clear that the nonlinearities in the Zebiak-Cane model suppress the growth of prediction errors caused by the FSVs, and the particular structure of the NFSVs reduces the suppression effect of nonlinearities, which finally results in the NFSVs to cause much larger prediction errors than the FSVs and represent the most disturbing tendency error.

\section{Summary and discussion}

In this study, we apply the NFSV approach to the ZebiakCane model to identify the most disturbing tendency error that has the largest effect on prediction uncertainties of El Niño events. For the predetermined reference-state El Niño events with different intensities, we calculate the corresponding NFSVs, which are superimposed to the SSTA equation of the model and act as the most disturbing tendency errors associated with for El Niño predictions. The results show that only one NFSV exists for each El Niño 

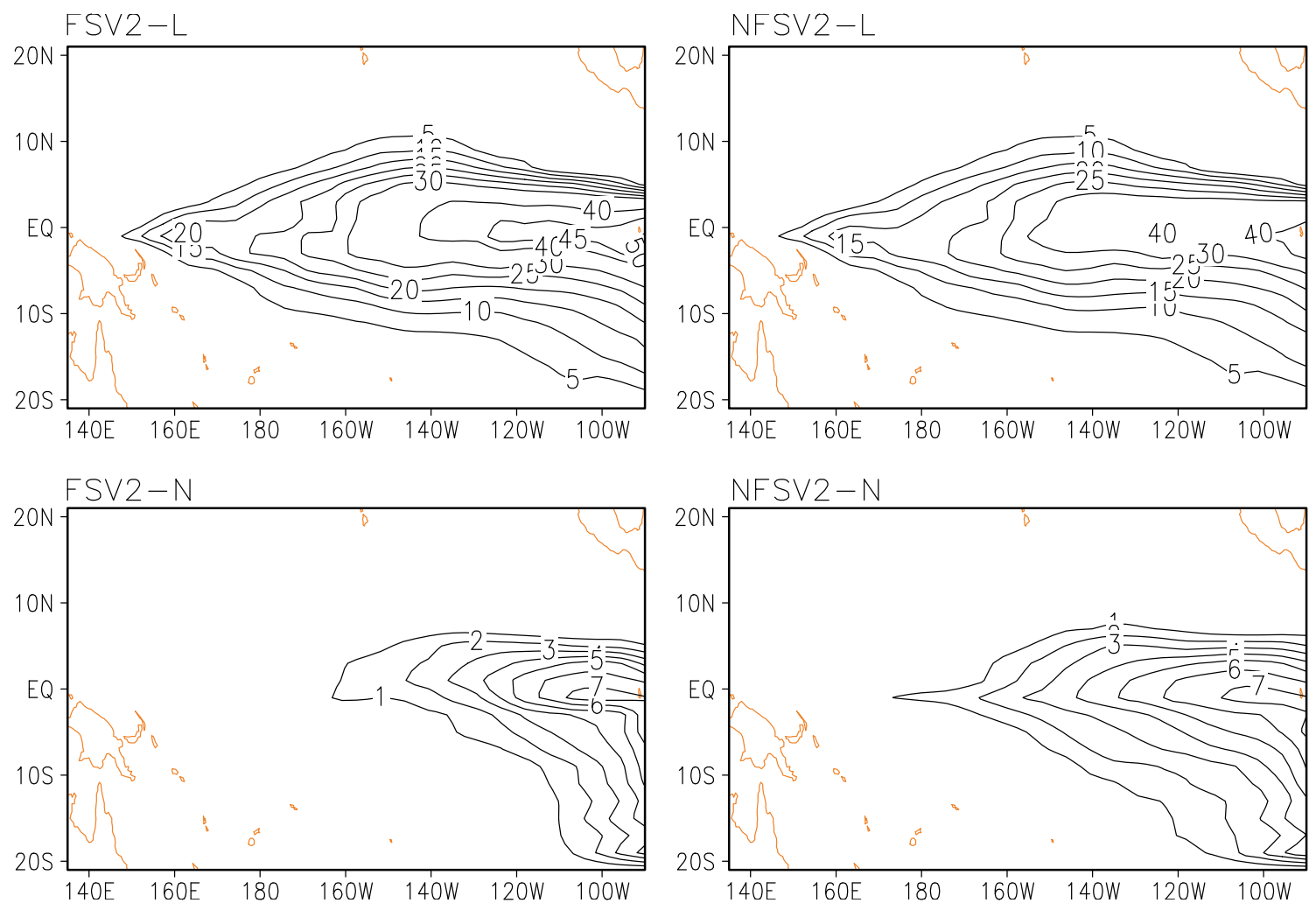

Fig. 8 As in Fig. 7, but for NFSV2 and FSV2

event prediction. These NFSVs always locate the boundary of the constraint conditions and often exhibit large-scale zonal dipolar patterns. For a particular start time of predictions, the dipolar patterns of NFSVs are insensitive to the intensities of El Niño events, but dependent on the prediction periods. Specifically, the NFSVs associated with the predictions crossing though the growth phase of El Niño are of a zonal dipolar pattern, with the western poles of positive anomalies in the equatorial central-western Pacific and the eastern poles of negative anomalies in the equatorial eastern Pacific. We denoted these as "NFSV1" tendency errors. Meanwhile, the NFSVs associated with the predictions crossing through the decaying phase of El Niño tend to have another zonal dipolar pattern, which is almost opposite to the NFSV1, which we refer to as "NFSV2" tendency errors. As a comparison, we also explore the FSVs, i.e., the linear counterpart of the NFSVs. The results show that the FSVs, similar to the NFSVs, can also be classified into two types. Correspondingly, the FSVs with the same signs as NFSV1 are denoted as "FSV1", and the other FSVs with the same signs as NFSV2 are referred to as "FSV2". The dipolar patterns of both FSV1 and NFSV1 tend to increase the equatorial eastern cooling-western warming thermal contrast and favor strong easterly anomalies, which persist to force tropical Pacific SSTAs. Then, a persistent anomalous upwelling occurs in the equatorial eastern Pacific, ultimately suppressing the warming of El Niño events and yielding a negative prediction error of the Niño-3 SSTA for the growth-phase predictions of El Niño events. Conversely, the FSV2 and NFSV2, since they have patterns opposite to the NFSV1 and FSVs, often cause positive prediction errors for the decaying-phase predictions of El Niño events. As demonstrated through the context, the NFSVs are directly from the nonlinear model while the FSVs come from the linearized model. Differences between the NFSVs and FSVs certainly exist. Actually, due to the effect of nonlinearities, the NFSVs usually have the western pole of the zonal dipolar pattern much farther west and cover a much broader region. Furthermore, we show that the FSVs yield much larger prediction errors for the Niño-3 SSTA in the linearized model than in the nonlinear model, and then the nonlinearities have a suppression effect on the growth of the prediction errors caused by the FSVs. However, the particular structure of the NFSVs tends to reduce the suppression effect of nonlinearities on the growth of prediction errors and results in the NFSVs to cause much large prediction errors in the nonlinear model. Obviously, the NFSV approach is superior to the FSV one in demonstrating the effects of nonlinearity on El Niño predictability, and is much more applicable in describing the most disturbing tendency error that causes the largest prediction errors. 
(a1)

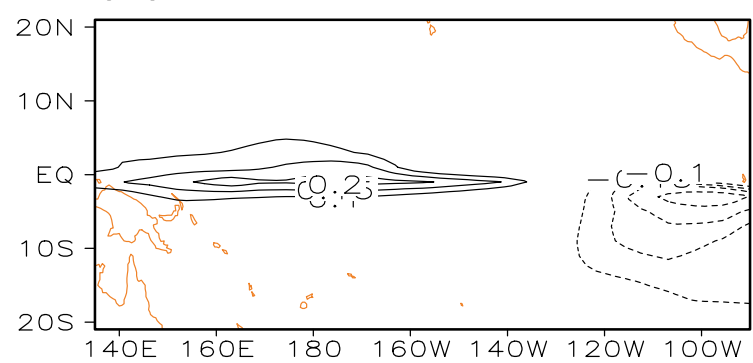

(b1)

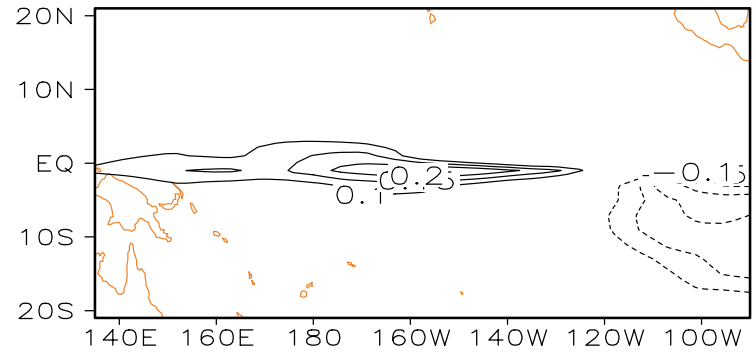

(c1)

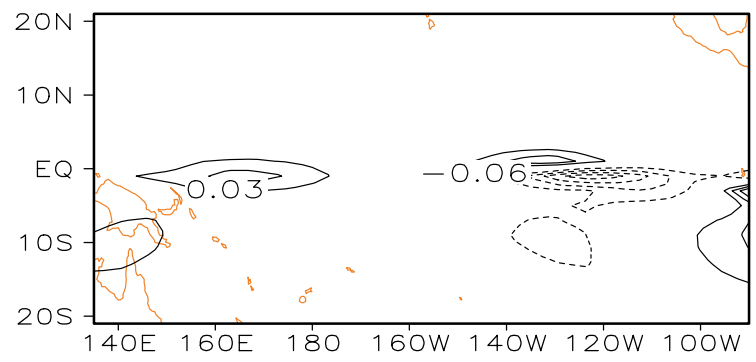

Fig. 9 The left column is for NFSV1 (a1), FSV1 (b1), and the difference between them (c1); the right column is for the NFSV2 (a2), FSV1 (b2), the difference between them (c2). The NFSV1 (NFSV2)

We notice that NFSVs and FSVs often concentrate the tendency errors of large values in few areas, which may indicate that the model errors in these areas have a much larger contribution to the occurrence of prediction errors. That is to say, the model errors in these few areas, compared to those in other areas, are much more likely to cause large prediction errors for El Niño events. These areas may represent the sensitive areas of El Niño predictions associated with model errors. Therefore, if the ability of the model simulating the states in the sensitive areas is improved, the ENSO forecast skill may in turn be greatly improved. The FSVs are derived from linearized models and act as an approximation to NFSVs (Duan and Zhou 2013). Correspondingly, due to the effect of nonlinearity, the sensitive areas identified by the NFSVs are somewhat different from those identified by the FSVs (see Sect. 4). Actually, the sensitive areas identified by the FSVs locate in the equatorial central-eastern Pacific and eastern Pacific, while those identified by the NFSVs are in the equatorial central-western (a2)

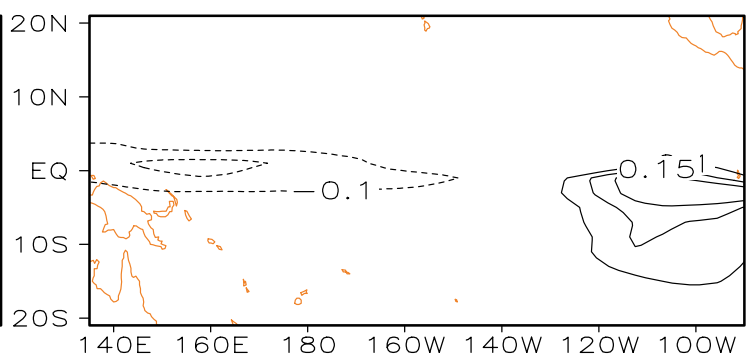

(b2)

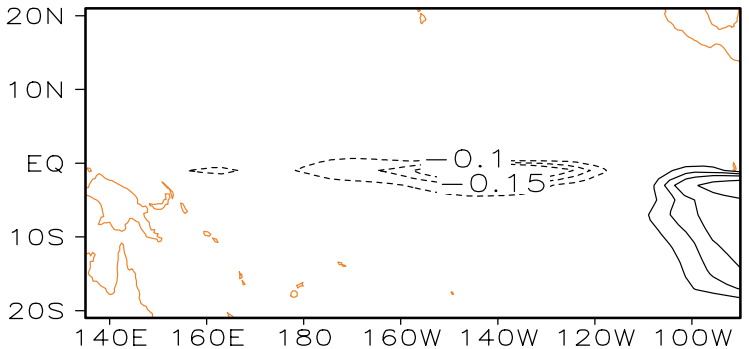

(c2)

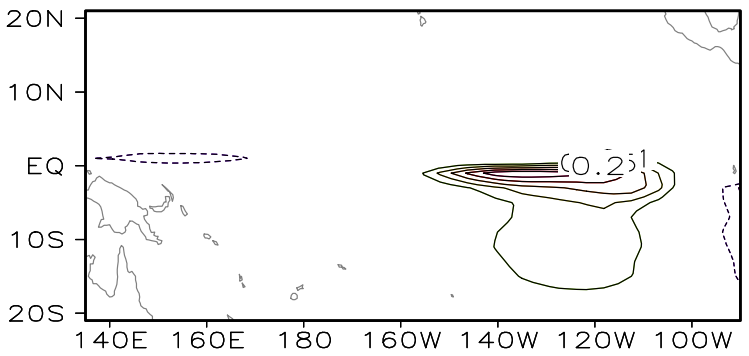

can be understood as the composite of the FSV1 (FSV2) and the difference between NFSV1 (NFSV2) and FSV1 (FSV2) (i.e. the additional SSTA tendency error of a dipolar pattern in the text)

Pacific and eastern Pacific. Considering the NFSVs are directly from the nonlinear model and do not have any approximation, we would rather believe that the NFSVs are much more applicable in identifying the sensitive areas associated with the model errors. That is to say, the sensitive areas identified by the NFSVs may be much more useful for improving the ENSO forecast skill. Of course, the validity of the sensitive areas identified by the NFSVs needs to be further validated by sensitivity experiments and even hindcast experiments, which we have begun to embark upon.

Predictability studies for ENSO are challenging due to the nonlinearity and complexity of the coupled oceanatmospheric system. In particular, the predictability problems associated with model errors pose great difficulties due to the lack of effective approaches. In this study, we use the NFSV approach to address the effect of constant tendency errors on ENSO predictability and reveal the behavior of nonlinearity modulating the prediction error caused by the tendency errors, which confirms that the NFSVs, compared 
to the FSVs, comprise a much more applicable approach in describing the tendency error that has the largest effect on prediction error in the nonlinear model. To further validate these results, a much more realistic ENSO model should be adopted to investigate the NFSVs. In addition, we should also study the optimal time-variant tendency errors and their effect on prediction errors for El Niño events. It is expected that the results obtained from the present work can provide ideas for further investigation on tendency errors.

Acknowledgments The authors appreciate the reviewers very much for their valuable comments and suggestions. This work was jointly sponsored by the National Basic Research Program of China (No. 2012CB955202), the National Public Benefit (Meteorology) Research Foundation of China (No. GYHY201306018), and the National Natural Science Foundation of China (Nos. 41176013 and 41230420).Open Access This article is distributed under the terms of the Creative Commons Attribution License which permits any use, distribution, and reproduction in any medium, provided the original author(s) and the source are credited.

\section{Appendix}

Definition, physics, and computation of CNOP

The conditional nonlinear optimal perturbation (CNOP) is an initial perturbation that satisfies a given constraint and has the largest nonlinear evolution at the prediction time (described below). The CNOP approach is a natural generalization of the linear singular vector (LSV) approach to a nonlinear system.

Let $M_{t_{0}, t}$ be the propagator (i.e., the numerical model) of a nonlinear model from initial time $t_{0}$ to $t . u_{0}$ is an initial perturbation superimposed on the basic state $U(t)$, which is a solution to the nonlinear model and satisfies $U(t)=M_{t}\left(U_{0}\right)$, with $U_{0}$ being the initial value of basic state $U(t)$.

For a selected norm $\|\cdot\|$, an initial perturbation $u_{0 \delta}$ is defined as a CNOP if and only if

$J\left(u_{0 \delta}\right)=\max _{\left\|u_{0}\right\| \leq \delta}\left\|M_{t_{0}, t}\left(U_{0}+u_{0}\right)-M_{t_{0}, t}\left(U_{0}\right)\right\|$,

where $\left\|u_{0}\right\| \leq \delta$ is the initial constraint defined by the selected norm $\|\cdot\|$. The norm $\|\cdot\|$ also measures the evolution of the perturbations. We can also investigate situations in which the initial perturbations belong to other types of functional sets. Furthermore, the constraint condition could reflect physical laws that the initial perturbation should satisfy.

The CNOP is characterized by maximum nonlinear evolution of initial perturbations satisfying the given constraint condition ( $\mathrm{Mu}$ et al. 2003; Mu and Zhang 2006). The CNOP possesses clear physical meanings (Duan and Mu 2009). Duan et al. (2004) demonstrated that when the objective function measures the maximum evolution of SSTAs for the ENSO, the resulting CNOP, superimposed on the climatological basic state, acts as the initial anomaly that is most likely to evolve into an El Niño event and represents the optimal precursor to El Niño. The CNOP can also be used to study the initial error with the largest effect on the prediction result at the prediction time (Duan and Mu 2009). In sensitivity analysis studies, the CNOP may represent the least stable mode and can be used to study target observations (Mu et al. 2009).

In the present study, we regard the CNOP as the optimal precursory disturbance (i.e., optimal initial anomaly) that is most likely to evolve into an El Niño event, and take the corresponding El Niño event as the reference state to explore the NFSV tendency errors. The CNOP-type initial anomalies, denoted by $u_{0 \sigma}$, are obtained by solving the following nonlinear optimization problem, which is based on Eq. (A.1):

$J\left(u_{0 \sigma}\right)=\max _{\left\|u_{0}\right\|_{\alpha} \leq \sigma}\|T(\tau)\|_{2}$

where $u_{0}=\left(w_{1}^{-1} T_{0}, w_{2}^{-1} h_{0}\right)$ is non-dimensional, denoting the initial SSTA and the thermocline depth anomalies superimposed on the climatological annual cycle. $w_{1}=2{ }^{\circ} \mathrm{C}$ and $w_{2}=50 \mathrm{~m}$ are the characteristic scales of SST and thermocline depth, respectively. $\left\|u_{0}\right\|_{\alpha} \leq \sigma$ is the constraint condition and the norm is $\left\|u_{0}\right\|_{\alpha}=\sqrt{\sum_{i, j}\left\{\left(w_{1}^{-1} T_{0 i, j}\right)^{2}+\left(w_{2}^{-1} h_{0 i, j}\right)^{2}\right\}}$, where $T_{0 i, j}$ and $h_{0 i, j}$ represent the dimensional initial SSTA and thermocline depth anomaly at different grid points, respectively, and $(i, j)$ is the grid point in the domain of the tropical Pacific (latitude and longitude, respectively), from $129.375^{\circ} \mathrm{E}$ to $84.375^{\circ} \mathrm{W}$ (at an interval of $5.625^{\circ}$ ) and from $19^{\circ} \mathrm{S}$ to $19^{\circ} \mathrm{N}$ (at an interval of $2^{\circ}$ ). The SSTA evolution is measured by $\|T(\tau)\|_{2}=\sqrt{\sum_{i, j}\left(T_{i, j}(\tau)\right)^{2}} . T(\tau)$ represents the SSTA component of the evolution of initial anomalies at time $\tau$, and is obtained by integrating the Zebiak-Cane model with initial anomalies.

We use the SPG2 solver to obtain the CNOP-type initial anomalies of the Zebiak-Cane model. To obtain CNOPtype initial anomalies, we modify the corresponding maximization problem into a minimization one and try at least 30 initial guesses (obtained randomly). If several initial guesses converge to a point in the phase space, this point can be considered a minimum in the neighborhood; thus, several such points are obtained, of which the one that yields the largest value of the cost function in Eq. (A.2) is regarded as the CNOP-type initial anomalies.

\section{References}

Barkmeijer J, Iversen T, Palmer TN (2003) Forcing singular vector and other sensitivity model structures. Q J R Meteorol Soc 129:2401-2423 
Birgin EG, Martínez JM, Raydan M (2000) Nonmonotone spectral projected gradient methods on convex sets. SIAM J Optim 10:1196-1211

Blanke B, Neelin JD, Gutzler D (1997) Estimating the effect of stochastic wind stress forcing on ENSO irregularity. J Clim 10(7):1473-1486

Blumenthal MB (1991) Predictability of a coupled ocean-atmosphere model. J Clim 4(8):766-784

Chen D, Zebiak SE, Busalacchi AJ, Cane MA (1995) An improved procedure for El Niño forecasting: implications for predictability. Science 269:1699-1702

Chen D, Cane MA, Kaplan A, Zebiak SE, Huang DJ (2004) Predictability of El Niño over the past 148 years. Nature 428:733-736

Duan WS, Mu M (2009) Conditional nonlinear optimal perturbation: applications to stability, sensitivity, and predictability. Sci China D 52:884-906

Duan WS, Zhang R (2010) Is model parameter error related to a significant spring predictability barrier for El Niño events? Results from a theoretical model. Adv Atmos Sci 27(5):1003-1013

Duan WS, Zhou F (2013) Non-linear forcing singular vector of a twodimensional quasi-geostrophic model. Tellus A 65. doi:10.3402/ tellusa.v65i0.18452

Duan WS, Mu M, Wang B (2004) Conditional nonlinear optimal perturbation as the optimal precursors for ENSO events. J Geophys Res 109:D23105

Duan WS, Xu H, Mu M (2008) Decisive role of nonlinear temperature advection in El Niño and La Niña amplitude asymmetry. J Geophys Res 113:C01014. doi:10.1029/2006JC003974

Duan WS, Liu XC, Zhu KY, Mu M (2009) Exploring the characteristic of initial errors that cause a significant "spring predictability barrier" for El Niño events. J Geophys Res 114:C04022. doi:10.1 029/2008JC004925

Duan WS, Yu Y, Xu H, Zhao P (2012) Behaviors of nonlinearities modulating the El Niño events induced by optimal precursory disturbances. Clim Dyn. doi:10.1007/s00382-012-1557-z

Flügel M, Chang P (1998) Does the predictability of ENSO depend on the seasonal cycle? J Atmos Sci 55(21):3230-3243

Gebbie G, Eisenman I, Wittenberg A, Tziperman E (2007) Modulation of westerly wind bursts by sea surface temperature: a semistochastic feedback for ENSO. J Atmos Sci 64(9):3281-3295

Hao Z, Ghil M (1994) Data assimilation in a simple tropical ocean model with wind stress errors. J Phys Oceanogr 24(10):2111-2128

Jin EK, James L, Kinter III, Wang B, Park C-K, Kang I-S, Kirtman BP, Kug J-S, Kumar A, Luo J-J, Schemm J, Shukla J, Yamagata T (2008) Current status of ENSO prediction skill in coupled oceanatmosphere models. Clim Dyn 31(6):647-664

Kleeman R (1991) A simple model of the atmospheric response to ENSO sea surface temperature anomalies. J Atmos Sci 48(1):3-19

Latif M, Sterl A, Maier-Reimer E, Junge MM (1993) Structure and predictability of the El Niño/Southern Oscillation phenomenon in a coupled ocean-atmosphere general circulation model. J Clim 6(4):700-708

Latif M, Anderson D, Barnett T, Cane M, Kleeman R, Leetmaa A, O'Brien J, Rosati A, Schneider E (1998) A review of the predictability and prediction of ENSO. J Geophys Res Oceans (19782012) 103(C7):14375-14393

Liu Z (2002) A simple model study of ENSO suppression by external periodic forcing. J Clim 15(9):1088-1098

Liu DC, Nocedal J (1989) On the limited memory method for large scale optimization. Math Program 45B:503-528

Lorenz EN (1996) Predictability-a problem partly solved. In: Proceedings of the ECMWF seminar on predictability, September 4-8, 1995, Reading, England, vol. 1. ECMWF, Shinfield Park, Reading, England, pp 1-18

Luo JJ, Masson S, Behera SK, Yamagata T (2008) Extended ENSO predictions using a fully coupled ocean-atmosphere model. J Clim 21(1):84-93
Marshall AG, Alves O, Hendon HH (2009) A coupled GCM analysis of MJO activity at the onset of El Niño. J Atmos Sci 66(4): 966-983

McCreary JP, Anderson DLT (1991) An overview of coupled oceanatmosphere models of El Niño and the Southern Oscillation. J Geophys Res Oceans (1978-2012) 96(S01):3125-3150

McPhaden MJ, Zebiak SE, Glantz MH (2006) ENSO as an integrating concept in Earth science. Science 314(5806):1740-1745

Moore AM, Kleeman R (1996) The dynamics of error growth and predictability in a coupled model of ENSO. Q J R Meteorol Soc 122(534):1405-1446

Mu M, Zhang Z (2006) Conditional nonlinear optimal perturbations of a two-dimensional quasigeostrophic model. J Atmos Sci 63:1587-1604

Mu M, Duan WS, Jiacheng W (2002) The predictability problems in numerical weather and climate prediction. Adv Atmos Sci 19(2):191-204

Mu M, Duan WS, Wang B (2003) Conditional nonlinear optimal perturbation and its applications. Nonlinear Process Geophys 10:493-501

Mu M, Xu H, Duan WS (2007a) A kind of initial perturbations related to "spring predictability barrier" for El Niño events in ZebiakCane model. Geophys Res Lett 34:L03709. doi:10.1029/200 6GL-27412

Mu M, Duan WS, Wang B (2007b) Season-dependent dynamics of nonlinear optimal error growth and El Niño-Southern Oscillation predictability in a theoretical model. J Geophys Res 112:D10113. doi:10.1029/2005JD006981

Mu M, Zhou F, Wang H (2009) A method to identify the sensitive areas in targeting for tropical cyclone prediction: conditional nonlinear optimal perturbation. Mon Weather Rev 137:1623-1639

Neelin JD (1990) A hybrid coupled general circulation model for El Niño studies. J Atmos Sci 47(5):674-693

Palmer TN, Andersen U, Cantelaube P et al (2004) Development of a European multi-model ensemble system for seasonal to inter-annual prediction (DEMETER). Bull Am Meteorol Soc 85(6):853-872

Penland C, Magorian T (1993) Prediction of Niño-3 sea surface temperatures using linear inverse modeling. J Clim 6(6):1067-1076

Powell MJD (1983) VMCWD: a Fortran subroutine for constrained optimization. ACM SIGMAP Bull 4-16. doi:10.1145/1111272.1111273

Roads JO (1987) Predictability in the extended range. J Atmos Sci 44:1228-1251

Saha S, Nadiga S, Thiaw C et al (2006) The NCEP climate forecast system. J Clim 19(15):3483-3517

Samelson RM, Tziperman E (2001) Instability of the chaotic ENSO: the growth-phase predictability barrier. J Atmos Sci 58(23): 3613-3625

Syu HH, Neelin JD (2000) ENSO in a hybrid coupled model. Part I: sensitivity to physical parametrizations. Clim Dyn 16(1):19-34

Tang Y, Yu B (2008) MJO and its relationship to ENSO. J Geophys Res 113:D14106. doi:10.1029/2007JD009230

Tang Y, Kleeman R, Moore AM (2008) Comparison of informationbased measures of forecast uncertainty in ensemble ENSO prediction. J Clim 21(2):230-247

Williams PD (2005) Modelling climate change: the role of unresolved processes. Philos Trans R Soc Math Phys Eng Sci 363(1837): 2931-2946

Wu DH, Anderson DLT, Davey MK (1993) ENSO variability and external impacts. J Clim 6(9):1703-1717

Xue Y, Cane MA, Zebiak SE, Blumenthal MB (1994) On the prediction of ENSO: a study with a low order Markov model. Tellus 46A:512-528

Yu Y, Mu M, Duan WS (2012) Does model parameter error cause a significant "Spring Predictability Barrier" for El Niño events in the Zebiak-Cane Model? J Clim 25(4):1263-1277 
Zavala-Garay J, Moore AM, Kleeman R (2004) Influence of stochastic forcing on ENSO prediction. J Geophys Res Oceans (19782012) 109:C11007. doi:10.1029/2004JC002406

Zebiak SE (1986) Atmospheric convergence feedback in a simple model for El Niño. Mon Weather Rev 114(7):1263-1271
Zebiak SE, Cane A (1987) A model El Niño-Southern oscillation. Mon Weather Rev 115:2262-2278

Zhang RH, Zebiak SE, Kleeman R, Keenlyside N (2003) A new interpmediate coupled model for El Niño simulation and prediction. Geophys Res Lett 30(19). doi:10.1029/2003GL018010 